\title{
Publisher's Note: Calculation of Projected Bond-Orientational Order Parameters to Quantify Local Symmetries from Transmission Diffraction Data [Phys. Rev. Lett. 116, 205501 (2016)]
}

A. C. Y. Liu, R. F. Tabor, L. Bourgeois, M. D. de Jonge, S. T. Mudie, and T. C. Petersen (Received 2 June 2016; published 9 June 2016)

DOI: 10.1103/PhysRevLett.116.239902

This Letter was published online on 18 May 2016 with an error in the source listing of Ref. [22]. Reference [22] should read as "H. Tanaka, Eur. Phys. J. E Soft Matter 35, 113 (2012)." The reference has been corrected as of 3 June 2016. The reference is incorrect in the printed version of the journal. 